\title{
Importancia de la estadística aplicada para la toma de decisiones en marketing
}

\author{
Importance of applied statistics for maketing decision making
}

Diego Alonso Villegas Zamora

diegovilleg@gmail.com

https://orcid.org/0000-0002-6080-6394

Universidad Privada del Valle, Cochabamba-Bolivia
Artículo recibido en marzo2021

Arbitrado en abril 2021

Aceptado en mayo 2021

Publicado en julio 2021

\section{RESUMEN}

Este artículo de revisión bibliográfica tuvo por objetivo demostrar la utilidad de la aplicación de diferentes medidas estadísticas descriptivas en el área de marketing. El estudio se desarrolló en dos fases: primero, se revisó la literatura existente con relación a la importancia de la estadística, la realidad de su enseñanza en la academia y su aplicación en las organizaciones; segundo, tomando como herramienta el paquete estadístico SPSS, se analizó la utilidad de la estadística descriptiva aplicada a un ejemplo ficticio de toma de decisiones en marketing. Como resultado se encontró que la estadística descriptiva aplicada constituye una herramienta de valor para los principales ejecutivos de una empresa, pues pueden utilizarla como un recurso para la toma de decisiones en el marketing. Se concluye que es pertinente crear una cultura estadística en el interior de las organizaciones.

Palabras clave: Estadística aplicada; Investigación de mercados; Toma de decisiones; Gestión empresarial; Marketing

The objective of this bibliographic review article was to demonstrate the usefulness of applying different descriptive statistical measures in the marketing area. The study was developed in two phases: first, the existing literature was reviewed in relation to the importance of statistics, the reality of its teaching in academia and its application in organizations; second, using the SPSS statistical package as a tool, the usefulness of descriptive statistics applied to a fictitious example of decision-making in marketing was analyzed. As a result, it was found that the descriptive statistics applied constitutes a valuable tool for the main executives of a company, as they can use it as a resource for decision-making in marketing. It is concluded that it is pertinent to create a statistical culture within organizations.

Key words: Applied Statistics; Market research; Decision making; Business management; Marketing 


\section{INTRODUCCIÓN}

La revolución tecnológica permite que la información sea cada vez de más fácil acceso para la colectividad (Rowley \& Farrow, 2018). A esta realidad se enfrentan las empresas actualmente, al contar con clientes con mayor acceso a fuentes externas de información a la hora de adquirir un producto o servicio. Sin embargo, no se debe perder de vista que el acceso a la información también constituye una ventaja para las empresas que, de manera oportuna, pueden valerse de ésta para la toma de decisiones.

Si bien el uso de la tecnología permite a las empresas contar cada vez con mayor cantidad de datos sobre los clientes, es cierto que pocas empresas cuentan con profesionales especializados en el análisis de datos para otorgar información oportuna y pertinente a las gerencias y al personal ejecutivo que les permita tomar decisiones en situaciones de menor incertidumbre (EasterbySmith et al, 2018). Por contradictorio que parezca, muchas veces el exceso de datos en una empresa constituye una especie de laberinto, en el cual los decisores no encuentran salida. Esto es en su mayoría debido a la carencia de personal especializado en el análisis de datos y a la falta de objetivos empresariales claros; en consecuencia, muchos ejecutivos se encuentran ante de datos que, si no son analizados debidamente, no contribuyen a la organización.

Una gran parte de la bibliografía en marketing considera la estadística descriptiva y de medidas estadísticas simples, como las de tendencia central, las de dispersión, las de localización, las de forma y las pruebast para muestras (Rowley \& Farrow, 2018). Sin embargo, es poca la literatura en marketing que profundiza en la utilidad que se puede dar a la estadística aplicada. La información con la que se cuenta sobre el uso de estos datos estadísticos de resumen descansa de manera general en un análisis teórico y genérico; sin embargo, el empleo la estadística en la toma de decisiones gerenciales vinculadas al marketing puede ser de bastante utilidad para disminuir la probabilidad de cometer errores.

Hasta el momento se ha hecho referencia a la gran cantidad de datos que genera de manera cotidiana una empresa. Sin embargo, no se debe perder de vista que el cliente constituye una fuente valiosa de información, la que no necesariamente estará en las bases de datos de la empresa, sino que deberá ser creada en función a las necesidades que se le presenten: medición de niveles de satisfacción, pruebas de conceptos, medición de niveles de lealtad, medición de percepciones, determinación de posicionamiento, posición competitiva, etc., es decir, información que provenga de investigaciones de mercado dirigidas a los clientes.

El diagnóstico de una organización de manera sistemática es una práctica estratégica de gran valor para una empresa. El marketing estratégico pivota esencialmente sobre la lectura clara que el estratega realice del mercado y todo su entorno; de este modo, el análisis de datos se constituye en un instrumento crítico a la hora de trabajar en el área estratégica de marketing. En este punto se puede aseverar la ponderación elevada que tiene el marketing estratégico con relación al marketing operativo a la hora de planificar las actividades de la organización a corto, mediano y largo plazo.

Con todo lo mencionado, este trabajo de revisión teórica se propuso demostrar la aplicación práctica de la interpretación de diferentes medidas estadísticas descriptivas en el área de marketing. Para este objetivo, el trabajo de investigación inicia con una revisión teórica sobre la importancia de la estadística aplicada en las organizaciones. En consecuencia, a partir del estado del arte, se pretende contar con la base suficiente para identificar su aplicación práctica en el área de marketing estratégico y operativo de una organización. 
MÉTODO

Con alcance descriptivo esta investigación tuvo como objetivo demostrar la utilidad de la aplicación de diferentes medidas estadísticas descriptivas en el área de marketing. El estudio se desarrolló en dos fases: primero, se revisó la literatura existente con relación a la importancia de la estadística, la realidad de su enseñanza en la academia, su aplicación en las organizaciones en general y en el marketing en particular; segundo, se analizó su utilidad mediante un ejemplo práctico.

Para la revisión bibliográfica se recurrió a trabajos de investigación publicados en repositorios digitales académicos, como también a fuentes bibliográficas de libros utilizados a nivel de pregrado como de postgrado. En todas estas obras se cuenta con las definiciones conceptuales y teóricas referentes a la estadística aplicada en las organizaciones.

Para el análisis y la demostración se presentó ejemplo hipotético de precios de vehículos que permitió mostrar a grandes rasgos cómo mediante la interpretación de estadísticos simples se otorga información valiosa a una empresa para la toma de decisiones en el área de marketing. El ejemplo se fundamenta en información hipotética obtenida a través de una investigación de mercados sobre cuánto estarían dispuestos a pagar las personas por un vehículo. Los estadísticos fueron obtenidos con el programa SPSS versión 24.

\section{RESULTADOS}

A continuación, se desarrollan los principales contenidos de la revisión bibliográfica realizada.

\section{Importancia de la estadística}

La enseñanza de la estadística ha cobrado relevancia desde hace varios años, debido a su importancia en la formación general del ciudadano (Batanero et al, 1994). La estadística involucra la vida de todas las personas, desde las encuestas de opinión hasta los ensayos clínicos. La sociedad actual genera nuevos espacios donde la metodología estadística adquiere un rol protagónico (Lent et al, 2002); de esta forma, el conocimiento de nociones básicas de estadística se torna importante al momento de entender la realidad (Sosa-Escudero, 2014). El reconocimiento de la estadística es incuestionable; sin embargo, su importancia no coincide con la importancia que se le da en la educación de los jóvenes (Sosa-Escudero, 2014). Si bien nociones estadísticas se han incluido en los diseños curriculares de la enseñanza secundaria, en general siguen estando ausentes; muchos profesores proponen el estudio de la estadística como último tema $\mathrm{y}$, cuando es posible, lo omiten (Azcárate, 2006).

Autores coinciden en que se debe entender que el conocimiento de la estadística es parte fundamental en la formación independientemente de la disciplina o profesión, es decir, que una cultura estadística permite a las personas en general comprender y utilizar con criterio el gran cúmulo de datos que existe (Azcárate, 2006; SosaEscudero, 2014; Lent et al, 2002). Actualmente, dado el desarrollo de la ciencia y tecnología, se hace también hincapié en cómo el análisis de los datos y su posterior interpretación estadística apoyan la toma de decisiones en cualquier área del conocimiento (Moreno, 1998; Batanero, 2002a; Utts, 2002).

En palabras de Muñoz (2007: 42) "En la sociedad actual, la estadística es un saber que deben poseer todos. [...] Sin perder su esencia adquiere la forma de la profesión que la contiene". A raíz de este papel fundamental en el cotidiano vivir, ha sido incorporada de forma generalizada como parte del currículo de Matemáticas, en las distintas carreras universitarias (Friz et al, 2011).

Behar y Ojeda (2000) expresan que todos los profesores deberían propender a una cultura estadística que comience en el colegio y se fortalezca en la universidad. Esta situación ha llevado a plantear como objetivos de la educación primaria 
y secundaria los siguientes: a) la comprensión y valoración de la estadística en la sociedad, incluyendo sus diferentes campos de aplicación; y b) la comprensión y valoración del método estadístico, dándole un uso inteligente a las formas básicas de razonamiento estadístico, su potencial y limitaciones (Batanero, 2002b).

Por su parte Holmes (1980), destaca una funcionalidad cívica que demanda la competencia de leer e interpretar tablas y gráficos que aparecen en los medios informativos, y considera que este saber es la base para el estudio de fenómenos complejos en distintas disciplinas. En esta misma dirección, Ottaviani (1998) señala que la estadística fomenta el desarrollo de valores y actitudes, como el razonamiento crítico y la valoración de evidencia objetiva, que permiten transformar datos para resolver problemas en situaciones de cotidianidad.

En función a todo lo anteriormente señalado, se concluye que varios de los autores citados coinciden en que la estadística, más allá de ser concebida como una materia en el currículo escolar o universitario, debe ser concebida como una asignatura de formación para la vida, independientemente del área de formación. Todo individuo debería contar con conocimientos mínimos de acopio, procesamiento y análisis de datos para, posteriormente, transformarlos en información que implique ayudar a la correcta toma de decisiones, ya sea de índole personal o institucional.

\section{Realidad de la enseñanza de la estadística}

Respecto a la enseñanza de la estadística, se precisa que la estadística tiene contenidos y procedimientos que le son propios y que, si no se trabajan de forma adecuada, no se interiorizan y se constituyen en barreras para que los estudiantes adquieran conocimientos más complejos. Según Batanero (2005), si esos contenidos no se trabajan debidamente se carece de bases sólidas que dificultan que los alumnos adquieran competencias que les permitan desenvolverse en la vida cotidiana con solvencia.

Estudios como el de Tovar et al. (2010) precisan, en sus resultados, que la enseñanza de la estadística en pregrado está concebida principalmente como cursos de cálculo matemático donde se limita al estudiante a realizar operaciones y cálculos matemáticos; en consecuencia, no se desarrolla un pensamiento analítico y de interpretación de los cálculos realizados de manera práctica en el área de formación en la cual se encuentra el estudiante.

En relación a este tema, estudios sobre libros o textos de matemáticas en la enseñanza de la estadística detectan que el proceso de enseñanzaaprendizaje se circunscribe a una gran cantidad de actividades repetitivas y que los libros de mayor uso abordan los contenidos temáticos de estadística descriptiva desde un enfoque procedimental, teniendo como falencia el objetivo de transmitir a los estudiantes la importancia del conocimiento estadístico para el manejo de la información diaria (Pomilio et al, 2017).

Se identifica como problema la permanencia del enfoque tradicional de enseñanza y aprendizaje, según expresa Muñoz (2007):

Por desgracia, los enfoques tradicionales de la enseñanza y el aprendizaje de la estadística, aún prevalentes en muchas escuelas, se ocupan sobre todo de promover la asimilación y repetición de definiciones tipo, de la ejecución eficiente y pulcra de algoritmos inalterables, del conocimiento y aplicación de fórmulas $\mathrm{y}$, en los niveles más avanzados, de la demostración formal de los fundamentos matemáticos de los conceptos. En este enfoque se supone que es posible aprender y comprender un concepto matemático o estadístico desligado del contexto en el que posteriormente habrá de ser transferido o aplicado e, incluso, esta descontextualización parece ser un requisito para lograr el aprendizaje de los conceptos (pág. 42). 


\section{Estadística aplicada}

Se puede definir la estadística aplicada como "un conjunto de procedimientos para reunir, medir, clasificar, codificar, computar, analizar y resumir información numérica adquirida sistemáticamente" (Ritchey, 2008, pag. 1). La utilidad de la estadística para el análisis en ciencias sociales se ha difundido bastante; para algunos autores, la estadística está concebida como la tecnología del método científico (Mood \& Craybill,1972).

La estadística aplicada puede considerarse como una disciplina pura, es decir, como una rama de las matemáticas, la cual se conoce, justamente, como estadística matemática, y como una disciplina aplicada, conocida como estadística aplicada (Pacheco, 2013). Cuando se habla de herramientas estadísticas aplicadas en la dirección de las organizaciones, se está haciendo referencia a la estadística aplicada. Desde luego, ésta no se reduce a la recolección de datos. Así, para Blalock (1998), la estadística comprende dos funciones: la primera, descripción de la información para facilitar su uso; la segunda, inducción para determinar población a partir de una muestra, formando así generalizaciones.

El campo de la estadística generalmente está dividido en dos grandes áreas que son la Estadística Descriptiva o Deductiva y Estadística Inferencial o Inductiva. La estadística descriptiva o deductiva es aquélla que se ocupa de describir y analizar un grupo determinado, sin que este estudio permita sacar conclusiones científicamente valederas de una muestra mayor. Se recolecta, presenta y caracteriza un conjunto de datos a fin de describir en forma apropiada las diversas características de éstos; es decir, un estudio se considera descriptivo cuando solamente se analizan y describen datos.

La estadística inductiva o inferencial es aquélla que se ocupa de inferir importantes conclusiones de una población a partir de una muestra representativa. Dicha inferencia no es del todo exacta; por esta razón, en este tipo de estadística se deben tomar en cuenta las probabilidades para establecer conclusiones. La inferencia estadística también se ocupa de estructurar una muestra representativa de la población en estudio, con el fin de diseñar modelos, inferencias, tendencias y predicciones sobre su comportamiento a partir de la aleatoriedad de las observaciones. Ese diseño se conseguiría empleando técnicas como la prueba de hipótesis, las estimaciones, la correlación, el análisis de regresión, las series de tiempo, la minería de datos, etc.

\section{Estadística en las organizaciones}

Las organizaciones sólidas dan importancia al tratamiento estadístico de la información relacionada, tanto con sus procesos internos como con el comportamiento de su entorno: políticas públicas, mercado, competidores, etc. (Pacheco, 2013). Particularmente, en áreas de economía y negocios: "La información obtenida al reunir datos, analizarlos, presentarlos e interpretarlos proporciona a directivos, administradores y personas que deben tomar decisiones una mejor comprensión del negocio o entorno económico, permitiéndoles tomar mejores decisiones con base en mejor información" (Anderson et al, 2008, pag. $3)$.

Las organizaciones enfrentan diversas situaciones empresariales haciendo uso de técnicas estadísticas, transformando los datos en información valiosa del entorno $y$, por ende, tomar mejores decisiones. En función a lo establecido por Pacheco (2013), éstas son algunas de las situaciones diarias en las que la estadística aporta: control estadístico de los procesos; nivel de fallas en los equipos y sus frecuencias; tiempos para cambios o preparación del herramental; niveles de productividad de distintos procesos; niveles de satisfacción de los clientes y usuarios; tipos de accidentes y sus frecuencias; análisis de los defectos y retrabajos; pronósticos de las ventas; comportamiento de los inventarios; nivel de 
cumplimiento de los proveedores; evolución de los distintos índices macroeconómicos y financieros; investigación de mercado; cálculo de costos y; desempeño del personal.

Así, la obtención de información completa, oportuna y confiable a través de las herramientas estadísticas se vuelve imprescindible para la dirección integral de las organizaciones. Al respecto, DeGroot y Fienberg (1981) afirma que la ciencia estadística se ocupa del desarrollo de teorías y técnicas apropiadas para realizar inferencias bajo condiciones de incertidumbre e ignorancia parcial, que inevitablemente existen en una amplia gama de actividades.

Se puede decir que, prácticamente, todas las herramientas de la estadística aplicada se adecuan a situaciones organizacionales con el fin de apoyar la toma de decisiones directivas; sin embargo, hay algunas cuya aplicación es más común (Webster \& García, 2000; DeGroot, 2012). A continuación, se presentan algunas de las más conocidas y utilizadas (Casas-Sánchez \& Santos-Peñas, 2002): medidas de tendencia central (media, mediana y moda); medidas de dispersión (varianza); medidas de posición o localización (cuartiles, deciles y percentiles); medidas de forma de la curva de distribución de frecuencias (sesgo y curtosis).

\section{Estadística en marketing}

Se ha establecido en párrafos precedentes lo útil que resulta la estadística aplicada en las organizaciones; consecuentemente, su utilidad en el área de marketing no escapa a esta realidad; muchas decisiones emergentes de estudios de mercado se pueden ver fortalecidas por el uso adecuado de la estadística. Sin embargo, no se debe perder de vista que, en la medida que se pueda obtener información en función a variables cuantitativas, el uso de la estadística será mucho más enriquecedora como información hacia los ejecutivos para la toma de decisiones.
La información constituye la base del marketing; esta se define como el conjunto de datos que tienen el potencial de influir en las decisiones de la gestión. En la medida en que los responsables de marketing no pueden controlar todos los factores de una situación dada, existe riesgo de que hagan una elección errónea; tales elecciones cuestan dinero (Gómez, 2013).

En el campo de la investigación de mercados, la estadística es de gran ayuda para la toma de decisiones; sin embargo, su utilidad estará estrechamente relacionada a qué objetivo persiga la empresa con la investigación de mercados; por ejemplo, en el momento de determinar qué tan probable es que un producto nuevo sea exitoso (Webster \& García, 2000), la estadística puede aportar en gran medida a describir las tendencias y preferencias del mercado en relación con el nuevo producto.

\section{Utilidad (demostración)}

De todo el análisis bibliográfico realizado se establece que existe un consenso generalizado por parte de la comunidad académica en que la formación de las personas en el área de estadística es fundamental como parte de habilidades y capacidades que se deben desarrollar, independientemente del área de formación de las personas. Por otrolado, la aplicación de la estadística como disciplina en el área de marketing descansa mayormente en temas de estudios académicos y no tanto en aspectos de orden práctico y objetivo para las organizaciones.

También se puede señalar que el empleo de la estadística se circunscribe al orden univariado donde el uso de distribuciones de frecuencias, gráficos, porcentajes, histogramas se constituyen en el principal recurso o herramienta para trabajar los datos y convertirlos en información que ayude a la toma de decisiones en la empresa. Este análisis, por cierto, es superficial, más aún si no se relacionan 
variables o se trabaja al menos con estadística bivariada a nivel de cruce de variables (tablas de contingencia).

La estadística en la investigación de mercados se utiliza mucho como recurso inferencial. Al trabajar con muestras representativas de poblaciones, muchos investigadores extrapolan sus hallazgos a lo que teóricamente quisiera toda la población; sin embargo, se debe tomar en cuenta que muchas de las inferencias realizadas no siguen con rigurosidad los aspectos metodológicos indispensables para que dichas inferencias sean confiables. Esto, como se advirtió en la revisión bibliográfica, obedece a una debilidad en la formación de los profesionales en lo relativo al empleo correcto de herramientas matemáticas dentro de la vida real y cotidiana (en este caso, poniendo el foco de atención en los problemas y retos a los que se enfrentan las organizaciones en cuanto a sus dilemas gerenciales).

Poseer información debidamente trabajada constituye una fuente de ventaja competitiva de difícil imitación por parte de los competidores; sin embargo, el contar con gente capacitada para realizar el análisis de manera objetiva y contextualizada es el reto que se hace difícil para las organizaciones. No se debe olvidar que la obtención de datos y la posterior generación de información para la toma de decisiones debe obedecer a que las empresas tengan claridad de los dilemas gerenciales en los que se encuentran y a los que posiblemente se enfrentará a futuro, además de que puedan tener la capacidad de transformar estos dilemas en protocolos claros y sistemáticos que guíen los procesos de investigación de manera objetiva.

A continuación, se presenta un ejemplo hipotético de precios de vehículos con el objetivo de mostrar a grandes rasgos cómo mediante la interpretación de estadísticos simples (tanto de forma aislada como en conjunto) se otorga información valiosa a una empresa para la toma de decisiones en el área de marketing. El ejemplo se fundamenta en información hipotética obtenida a través de una investigación de mercados sobre cuánto estarían dispuestas a pagar las personas por un vehículo. A continuación, se presentan los cuadros obtenidos con el programa SPSS versión 24 de acuerdo con una base de datos hipotética y transformada para fines didácticos.

Tabla 1. Estadístico de resumen.

\begin{tabular}{lccc}
\hline \multicolumn{1}{c}{ Estadísticos } & Hombres & \multicolumn{1}{l}{ Mujeres } \\
\hline Media & & $20301.40 \$$ & $13091.97 \$$ \\
$95 \%$ de intervalo de confianza para la media & Límite inferior & $19184.30 \$$ & $12698.26 \$$ \\
& Límite superior & $21418.49 \$$ & $13485.67 \$$ \\
Media recortada al 5\% & $19249.94 \$$ & $12849.56 \$$ \\
Mediana & $15750.00 \$$ & $12375.00 \$$ \\
Desv. Desviación & $9111.78 \$$ & $2935.60 \$$ \\
Mínimo & $9000.00 \$$ & $9000.00 \$$ \\
Máximo & $79980.00 \$$ & $30000.00 \$$ \\
Asimetría & 2.39 & 1.77 \\
Curtosis & 8.49 & 5.35 \\
\hline
\end{tabular}


Los datos de la Tabla 1 no se constituyen en información sino se los analizan e interpretan adecuadamente. Analizando la tabla, se puede advertir que existe, en promedio, una mayor disposición a pagar por un vehículo en los clientes hombres en relación con las clientes mujeres. La asimetría positiva en ambos casos indica que existe mayor concentración de los datos en valores bajos y que la curva de distribución de frecuencias para el caso de los varones está un tanto más dispersa en relación con las féminas $(2,39$ y 1,77, respectivamente). Gráficamente, se comprueba esta situación mediante la observación de los histogramas (ver figuras 1 y 2 ).

Aplicando una prueba $\mathrm{t}$ de diferencia de medias, se advierte que la diferencia de los dos promedios, desde el punto de vista de la estadística, es significativa, ya que el valor de contraste obtenido en la prueba es menor al nivel de significancia de 0.05: por lo tanto, se rechaza la hipótesis nula de que los dos promedios son iguales (ver Tabla 2).

Tabla 2. Prueba t para muestras no relacionadas.

\begin{tabular}{|c|c|c|c|c|c|c|}
\hline \multirow{3}{*}{$\begin{array}{c}\text { Hipótesis } \\
\\
\text { Se asumen } \\
\text { varianzas } \\
\text { iguales }\end{array}$} & \multicolumn{2}{|c|}{$\begin{array}{c}\text { Prueba de Levene de igualdad } \\
\text { de varianzas }\end{array}$} & \multicolumn{4}{|c|}{ Prueba t para la igualdad de medias } \\
\hline & $\mathrm{F}$ & Sig. & $\mathrm{t}$ & gl & Sig. (bilateral) & $\begin{array}{l}\text { Diferencia de } \\
\text { medias }\end{array}$ \\
\hline & 105.97 & $0.00<0.05$ & 11.15 & 472.00 & 0.00 & 7209.43 \\
\hline
\end{tabular}

Según la información presentada en la Tabla 2 se rechaza la hipótesis nula. El precisar que la diferencia de los promedios de disposición de pago entre hombres y mujeres es estadísticamente significativa y diferente tiene una connotación de decisiones ejecutivas bastante complejas.

Por ejemplo: (a) Se tiene información para planificar las campañas promocionales de los vehículos que produzca la empresa con un mensaje y publicidad diferentes, es decir, campañas publicitarias dirigidas a cada uno de los segmentos. Se advierte que los hombres son menos sensibles al precio cuando se trata de la compra de un vehículo; sin embargo, las mujeres son mucho más sensibles a la variable precio y no parecen estar dispuestas a pagar mucho por un vehículo. Esto es algo que se refrenda con los máximos a pagar por cada uno de los segmentos (79 980 \$ para hombres y 30000 \$ para mujeres); (b) Sugiere lanzar campañas publicitarias que resalten con mayor fuerza ciertos atributos de los vehículos; sobre todo, aquellos que se consideren como importantes para las mujeres, como seguridad, familiar, económico, etc., es decir, que la decisión de los ejecutivos también podría encaminarse por trabajar campañas comunicacionales únicas; (c) Especulando un poco más, el análisis de los promedios puede tener su efecto en la variable producto, ya que los ejecutivos de la empresa pueden optar también por desarrollar diferentes gamas de productos para alcanzar y satisfacer a los diferentes segmentos de mercado, que en este caso están denotados por la variable sexo; (d) El análisis de la desviación típica, conjuntamente al de la asimetría, puede sugerir a los ejecutivos que la compra de vehículos está influenciada en los hombres de manera más emocional en relación con las mujeres, que parecen ser más racionales en cuanto a la predisposición de adquirir un vehículo. Los valores en el caso de los varones están más dispersos. 
Tabla 3. Medidas de localización o posición.

\begin{tabular}{cccccccc}
\hline Género & \multicolumn{7}{c}{ Percentiles } \\
& $\mathbf{5}$ & $\mathbf{1 0}$ & $\mathbf{2 5}$ & $\mathbf{5 0}$ & $\mathbf{7 5}$ & $\mathbf{9 0}$ & $\mathbf{9 5}$ \\
\hline Hombre & 13500 & 14100 & 15000 & 15750 & 22688 & 33000 & 36788 \\
Mujer & 10178 & 10200 & 11131 & 12375 & 14250 & 17643 & 18750 \\
\hline
\end{tabular}

Los percentiles de la Tabla 3, al ser medidas de posición, otorgan información concerniente a la localización de las disposiciones a pagar por parte de los clientes. Aplicando la interpretación de los percentiles al marketing, se concluye lo siguiente: (a) Para el caso de los hombres, tan solo un $5 \%$ del mercado estaría dispuesto a pagar por un vehículo más de 36788 \$; en el caso de las mujeres, tan solo un 5\% del mercado estaría dispuesto a pagar más de 18750 \$. Este análisis obedece al percentil 95\%; (b) Cruzando el percentil 75 con el valor mínimo obtenido para cada grupo en los estadísticos de resumen, se tiene el siguiente análisis: para el caso de los hombres, el $75 \%$ del mercado estaría dispuesto a pagar entre 9000 \$ y 22688 \$; para el caso de las mujeres, el 75\% del mercado estaría dispuesto a pagar entre 9000 \$ y 14250 \$.

Se advierte que los percentiles son una fuente de información valiosa para cuantificar el posible mercado de referencia. Los percentiles pueden ayudar mucho a los decisores en temas relacionados a segmentación de mercados con base a una variable cuantitativa.
Suponiendo, por un momento, que la empresa comercializa vehículos dirigidos al segmento de hombres y que el precio mínimo de venta de uno de sus vehículos es de 30000 \$; con esta información y con base en los resultados de la Tabla 2, se concluye que la empresa tan solo estaría compitiendo en un mercado del $10 \%$, ya que el percentil 90 con un valor de 33000 \$ muestra esa realidad. No obstante, el hablar de competir en un $10 \%$ del mercado no necesariamente es despreciable de acuerdo con el contexto (tamaño de la población).

Con base en los percentiles una empresa dedicada a la venta de vehículos podría definir su estrategia competitiva, optando quizás por la diferenciación, el enfoque o tal vez el liderazgo en costos. Claramente, existen empresas comercializadoras de vehículos que optan por alguna de las estrategias genéricas anteriormente mencionadas y establecidas en la teoría por Michael Porter en 1982 (Porter, 2015).

Los histogramas (Figuras 1 y 2 ), como se mencionó anteriormente, muestran el comportamiento de los datos de forma gráfica.

Figura 1. Histograma de distribución de frecuencias - hombres.

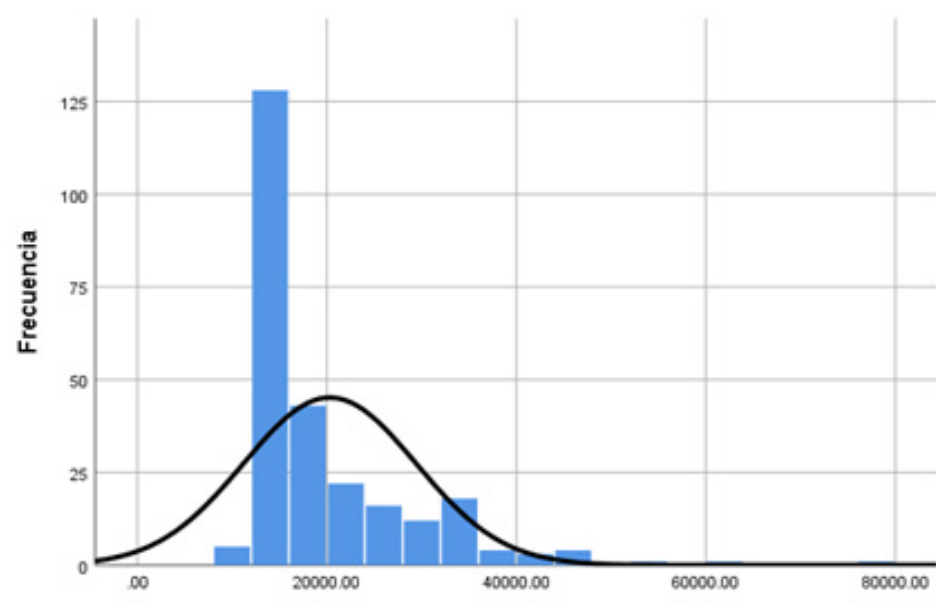


Figura 2. Histograma de distribución de frecuencias - mujeres.

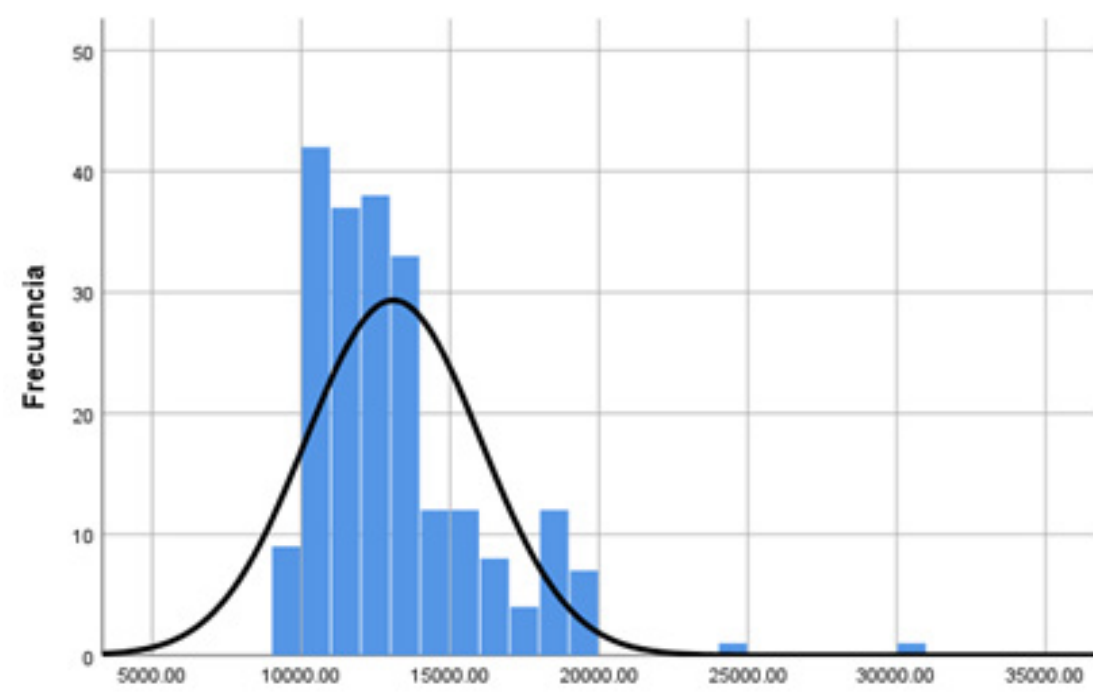

Analizando los histogramas (Figuras 1 y 2), se refrendan los análisis de la desviación típica y la asimetría. Se advierte cómo la distribución de frecuencias para el caso de los hombres está más dispersa en relación con la de las mujeres; además, la asimetría, al ser más alta en el caso de los hombres (2.39), refleja mayor concentración en precios bajos. Toda empresa esperaría obtener asimetrías negativas para la variable disposición a pagar por el producto, ya que concluiría que las disposiciones de pago de los clientes se encuentran concentrados en valores altos (precios altos).

\section{CONCLUSIONES}

Muchas de las soluciones en el mundo laboral van de la mano del uso de herramientas de análisis cuantitativo. Aquellos profesionales que no hayan desarrollado capacidades y habilidades en el análisis estadístico de problemas comunes en las empresas tendrán, sin duda, debilidades y desventajas en los mercados laborales frente a profesionales que cuenten con una cultura estadística.

No se puede asegurar que el análisis cuantitativo en las decisiones de marketing garantice el éxito; sin embargo, el uso de herramientas estadísticas aplicadas objetivamente al análisis de datos se constituye en una importante fuente de información para los tomadores de decisiones y, por ende, en una herramienta que se traduce en una ventaja competitiva de difícil imitación.

La interpretación de las diferentes medidas estadísticas debe obedecer a un análisis en conjunto y cruzado de los resultados obtenidos; un estudio aislado de los estadísticos de resumen no otorgaría la claridad requerida para poder guiar a los ejecutivos de una empresa en la toma de decisiones.

Es importante precisar en el lector que el uso de todas las herramientas estadísticas a disposición de los analistas debe responder a un uso adecuado de los supuestos que muchos análisis estadísticos precisan para ser válidos (distribución normal de los datos, muestras representativas, inferencia estadística, muestreo, etc.). Si se respetan los supuestos teóricos estadísticos, la aplicación práctica será de mucha utilidad.

Existen muchos más recursos estadísticos de los cuales podría valerse un analista de datos en una organización para otorgar información substancial a la parte ejecutiva. El presente trabajo de reflexión teórica solo pretende, con ejemplos básicos y sencillos, demostrar la utilidad de aplicar estas herramientas de manera práctica en la vida cotidiana y laboral. 
REFERENCIAS

Anderson, D.; Sweeney, D. \& Williams, T (2008). Estadística para administración y economía. 0 ed. México. Cengage Learning

Azcárate, P. (2006). ¿Por qué no nos gusta enseñar estadística y probabilidad? Conferencia realizada en XII, Jornadas de Investigación en el Aula de Matemáticas: Estadística y azar, Granada, España

Batanero, C. (2002a). Los retos de la cultura estadística, Jornadas interamericanas de enseñanza de la estadística, Buenos Aires, conferencia inaugural

Batanero, C. (2002b). Estadística y didáctica de la matemática: Relaciones, problemas y aportaciones mutuas. En M. Penalva, G. Torregrosa y J. Valls (Coords.). Aportaciones de la didáctica de la Matemática a diferentes perfiles profesionales (pp. 95-120). Murcia: Editorial Compobell

Batanero, C. (2005). Significados de la probabilidad en la educación secundaria. Revista Latinoamericana de Investigación en Matemáticas, 8(3), 247-264

Batanero, C., Godino, J. D., Green, D. R., Holmes, P \& Vallecillos, A. (1994). Errors and difficulties in understanding elementary statistical concepts. International Journal of Mathematics Education in Science and Technology, 25(4), 527-547

Behar, R. \& Ojeda, M. (2000). El proceso de aprendizaje de la estadística: ¿Qué puede estar fallando? Heurística, 10, 26-43

Blalock, H. M. (1998). Estadística social. México. FCE. 6" reimpresión

Casas-Sánchez, J. M. \& Santos-Peñas, J. (2002). Introducción ala estadística para administración y dirección de empresas segunda edición

DeGroot, M. H. (2012). Probability and statistics. Pearson

DeGroot, M. H., \& Fienberg, S. E. (1981). Assessing Probability Assessors: Calibration and Refinement. CARNEGIE-MELLON UNIV PITTSBURGH PA DEPT OF STATISTICS
Easterby-Smith, M., Thorpe, R., Jackson, P. R., \& Jaspersen, L. J. (2018). Management and business research. Sage

Friz, C. M.; Sanhueza, H. S. \& Figueroa, M. E. (2011). Concepciones de los estudiantes para profesor de Matemáticas sobre las competencias profesionales implicadas en la enseñanza de la Estadística. Revista electrónica de investigación educativa, 13(2), 113-131

Gómez, L. (2013). Diseño de un sistema de información de marketing. Tesis para optar por el título de Máster en Dirección, Universidad de La Habana. 2008. [2]. s/a, (2013). Los sistemas de Marketing, Recuperado el 2013, de cvh. ehu.es: http://cvb.ehu.es/open_course_ware/ castellano/social_juri /marketing/tema-5/ tema-5.pdf

Holmes, P. (1980). Teaching Statistics, 11-16. Sloug: Foulsham Educational

Lent, B., Dost, S., Redden, R. F., \& Liu, Y. (2002). Mathematical simulation of the traveling heater method growth of ternary semiconductor materials under suppressed gravity conditions. Journal of crystal growth, 237, 1876-1880

Mood, A.M. \& Graybill, E.A, (1972). Introducción a la teoría estadística. México

Moreno, J.L. (1998), "Statistical Literacy. Statistics Long after School", en L., Pereira- Mendoza et al. (ed.), Proceedings of the 5th International Conference on Teaching Statistics, Singapur, pp. $447-452$

Muñoz, E. D. (2007). El aprendizaje de la estadística en estudiantes universitarios de profesiones no matemáticas. Educación Matemática, 19(2), 4166

Ottaviani, M. G. (1998). Developments and perspectives in statisticaleducation. Documento presentado en el Congreso Estadística para el desarrollo Económico y Social, Aguascalientes, México

Pacheco, E. A. (2013). La estadística aplicada como herramienta para la dirección integral de las organizaciones. (Spanish). Gestión Y Estrategia, (44), 125-138 
Pomilio, C., Miño, M., Brignone, N., Facal, G., Telesnicki, M., Fass, M., Filloy, J., Cueto, G., Fernández, M., \& Perez, A. (2017). Análisis de actividades sobre estadística descriptiva en libros de educación media: ¿Qué se pretende que los estudiantes aprendan?

Porter, M.E. (2015). Estrategia competitiva: técnicas para el análisis de los sectores industriales y de la competencia. Grupo Editorial Patria

Ritchey, E. J. (2008). Estadística para las ciencias sociales. México. McGraw-Hill. 2da edición

Rowley, J., \& Farrow, J. (2018). Organizing Knowledge: An Introduction to Managing Access to Information: Introduction to Access to Information. Routledge

Sosa-Escudero, W. (2014). Qué es (y qué no es) la estadística: Usos y abusos de una Disciplina clave en la vida de los países y las personas. Buenos Aires: Siglo Veintiuno Editores
Tovar, C. J.; Castillo, S. H. \& Marín, M. del P. (2010). Preconcepciones de estudiantes de la Pontificia Universidad Javeriana Cali sobre el curso de estadística. Pensamiento Psicológico, 3(9). Recuperado a partir de //revistas.javerianacali. edu.co/index.php/pensamientopsicologico/ article/view/60

Utts, J. (2002), "What Educated Citizens Should Know About Statistics and Probability", Proceedings of the 6th International Conference on Teaching Statistics, Sudáfrica

Webster, A. L., \& García, Y. M. (2000). Estadística aplicada a los negocios y la economía 